\title{
Opioid Tapering and the Patient-Provider Relationship
}

\author{
Marianne S. Matthias, Ph.D. \\ VA HSR\&D Center for Health Information and Communication, Regenstrief Institute, Indiana University, Indianapolis, IN, USA.
}

$\mathrm{J}$ Gen Intern Med 35(1):8-9

DOI: $10.1007 / \mathrm{s} 11606-019-05337-4$

(c) Society of General Internal Medicine (This is a U.S. government work and not under copyright protection in the U.S.; foreign copyright protection may apply) 2019

$\mathrm{T}$ he surge in opioid prescribing in recent years has led to widely recognized harms and the declaration of a national opioid crisis in the USA. In response to this crisis, the Centers for Disease Control and Prevention (CDC) and the Department of Veterans Affairs/Department of Defense (VA/DoD) issued revised guidelines in 2016-2017. ${ }^{1,2}$ For patients on long-term opioid therapy for chronic pain, these guidelines recommend tapering to lower doses or discontinuation when benefits do not clearly outweigh potential harms. Despite these guidelines, there is insufficient evidence to support any particular tapering approach, and, more concerning, there is a dearth of information on patient outcomes during and after tapering. A 2017 systematic review by Frank and colleagues found that many studies reported positive outcomes from opioid dose reductions, including improvements in pain severity, function, and quality of life. ${ }^{3}$ However, the quality of this body of evidence was determined to be very low. Moreover, for the studies that showed positive outcomes, the interventions tended to involve team-based, intensive support for patients - a model that is guideline-concordant, but not always easily implemented, especially in busy primary care settings.

Given the paucity of evidence on outcomes of opioid tapering, the study by Perez and colleagues ${ }^{4}$ in this issue is significant and timely. This study explores an important yet overlooked aspect of potential consequences of opioid tapering: termination of care. These authors conducted a retrospective cohort study of patients on long-term opioid therapy for chronic non-cancer pain, using data from the electronic medical record. They identified a cohort of patients prescribed a stable dose of opioids (defined as varying by $<30 \%$ ) for at least a year (the baseline year), determined whether or not they experienced an opioid taper the next year (exposure year), and determined whether they terminated their care in the following year (the outcome year). To be included, patients had to have had three or more opioid prescriptions, at least 21 days apart, in two consecutive 6-month periods, at a dose of at least 25 morphine milligram equivalents (MMEs). In the absence of a standard definition for what constitutes an opioid taper, the authors defined a taper for their primary analysis as a

Published online November 8, 2019 reduction in average daily MME of at least $30 \%$ compared with baseline, in both 6-month periods during the exposure year. For secondary analyses, they examined reductions of at least $50 \%$. The authors compared the group who experienced tapering to a comparison group who experienced either a dose increase, no dose change, or no more than $30 \%$ decrease compared with their baseline dose ("continued" group).

Of 1624 patients identified, with a median dose of $90 \mathrm{MME}$ at baseline, 207 (12.7\%) patients had an opioid taper, 1131 had dose continuations, and the remainder were unspecified (i.e., the authors were unable to determine whether a change in dose was an intentional taper or simply a fluctuation in dose). During the outcome year, 78 patients (4.8\% of total sample) experienced termination of care. Of the 207 tapered patients, $18(8.7 \%)$ terminated care, versus 22 patients in the continued group (2\% of this group) and $38(13.3 \%)$ in the unspecified group. Analyses revealed that in the tapered versus continued groups, opioid taper was associated with more than four times the odds of termination of care (AOR 4.3 [95\% CI 2.2-8.5]). Results were similar using the $50 \%$ dose reduction to define a taper, with opioid taper associated with 3.8 times the odds of termination of care.

Perez and colleagues have identified termination of care as a potentially harmful unintended consequence of opioid tapering. As the authors point out, termination of care is associated with numerous poor health outcomes, and these patients may also be at risk for opioid misuse or overdose, as well as depression or suicidality if pain is uncontrolled. The authors acknowledge several limitations to their study, including the absence of information on the reason for the taper, and whether termination of care was a direct result of the tapering. In addition, they acknowledge that it is unclear how many patients underwent involuntary tapering, versus those who agreed with the tapering decision. This is likely an important factor that determines patient outcomes during and after tapering.

Despite these limitations, this study offers an important note of caution for the widespread opioid tapering that is occurring, in the absence of a strong body of evidence supporting particular tapering strategies or fully understanding patient outcomes after tapering. Moreover, these results underscore the need for effective patient-provider communication prior to initiation of tapering, so that patients understand why tapering is needed, and what the risks of opioids are to them personally. This latter point is especially important given that studies of patients' tapering experiences have found that, while patients 
generally understand the global risks of opioids, they tend not to believe that such risks apply to them. ${ }^{5}{ }^{6}$ Consequently, tapering these patients could result in dissatisfaction with care, ultimately leading to care termination. To mitigate this risk and enhance patient understanding of opioid tapering, strategies in which providers tailor their messages to a patient's particular health concerns and how opioids may exacerbate these health concerns (e.g., explaining to a patient with pulmonary disease that opioids may depress respiratory function) may be an important tool to gain patient buy-in to opioid tapering, which presumably could reduce the risk of termination of care. ${ }^{5}$

Another important consideration prior to and during tapering is patient input into the process. Patient involvement in treatment decisions has been shown to be associated with higher patient satisfaction, better treatment adherence, and, in some cases, better patient outcomes. ${ }^{7}$ As a result, facilitating patient participation in tapering decisions is likely to improve retention in care. While not all tapers are voluntary, even mandated tapers can be done in a way that gives patients a voice. For example, collaboratively negotiating the rate of tapering with a patient can go a long way toward giving a patient a sense of control over their pain management. ${ }^{5}$ Finally, fostering and maintaining a strong patient-provider relationship, which is always important in pain management, appears to be particularly important for opioid tapering. Patients have identified having a trusted, supportive provider as a key facilitator to successful tapering. ${ }^{5,6}$ This means more than just having a "good relationship"; in the context of tapering, this means that patients understand and trust that their provider is not going to cut their opioids and then abandon them. Tapering studies have found that both patients and providers understood the need for patients to feel as though their providers were supportive and accessible throughout the tapering process, particularly if patients experienced difficulties. ${ }^{5,6}$ In one study, providers even expressed the willingness to increase an opioid dose temporarily during tapering if the patient was having difficulty adjusting to a lower dose. ${ }^{5}$ Another study found that patients were more willing to accept opioid dose reductions if they believed their providers genuinely cared about them and their wellbeing. ${ }^{8}$ This flexibility and compassion is the cornerstone of a solid, trusting patientprovider relationship, and is critical to mitigate potential adverse outcomes of tapering, including termination of care.

Communication has long been identified as an integral part of pain management. In an era where patients are increasingly having their opioids reduced or discontinued, communication is even more critical. Unfortunately, communication has also been described as challenging in pain management - especially when opioids are concerned., 10 Tapering can exacerbate these communication challenges, especially when patients do not undergo tapering willingly. Optimizing outcomes of tapering means that, not only is more evidence needed on tapering strategies, but also that patients must feel a part of the process, understand why the taper is necessary for them personally, and feel supported and cared for by their providers.

Corresponding Author: Marianne S. Matthias, Ph.D.; VA HSR\&D Center for Health Information and Communication, Regenstrief InstituteIndiana University, Indianapolis, IN, USA (e-mail: mmatthia@iupui.edu).

\section{Compliance with Ethical Standards:}

Conflict of Interest: The author declares no conflict of interest.

\section{REFERENCES}

1. Dowell D, Haegerich TM, Chou R. CDC guideline for prescribing opioids for chronic pain-United States, 2016. Morb Mortal Wkly Rep. 2016;65(1): 1-49.

2. VA/DoD Opioid Therapy Work Group. Clinical Practice Guideline for Management of Opioid Therapy for Chronic Pain. Washington, D.C. 2017. https://www.healthquality.va.gov/guidelines/Pain/cot/. Accessed 4 Aug 2019.

3. Frank JW, Lovejoy TI, Becker WC, et al. Patient outcomes in dose reduction or discontinuation of long-term opioid therapy: a systematic review. Ann Intern Med. 2017;167:181-191.

4. Perez HR, Buonora M, Cunningham C, Moonseong $\mathbf{H}$, Starrels $\mathrm{JL}$ Opioid taper is associated with subsequent termination of care: a retrospective cohort study. J Gen Intern Med. 2019. https://doi.org/10. 1007/s11606-019-05227-9.

5. Matthias MS, Johnson N, Shields CG, et al. "I'm not gonna pull the rug out from under you": patient-provider communication about opioid tapering. J Pain. 2017;18(11):1365-1373.

6. Frank JW, Levy C, Matlock DD, et al. Patients' perspectives on tapering of chronic opioid therapy: a qualitative study. Pain Med. 2016;17:1838-1847.

7. Joosten EA, DeFuentes-Merrilas L, de Weert GH, Sensky T, van der Staak CP, de Jong CA. Systematic review of the effects of shared decision-making on patient satisfaction, treatment adherence, and health status. Psychother Psychosom. 2008;77(4):219-226.

8. Matthias MS, Krebs EE, Bergman AA, Coffing JM, Bair MJ. Communicating about opioids for chronic pain: A qualitative study of patient attributions and the influence of the patient-physician relationship. Eur J Pain. 2014; 18:835-843.

9. Upshur CC, Bacigalupe G, Luckmann R. "They don't want anything to do with you": patient views of primary care management of chronic pain. Pain Med. 2010;11(12):1791-1798.

10. Matthias MS, Parpart AL, Nyland KA, et al. The patient-provider relationship in chronic pain care: providers' perspectives. Pain Med. 2010;11:1688-1697.

Publisher's Note Springer Nature remains neutral with regard to jurisdictional claims in published maps and institutional affiliations. 